\title{
Rumination, Negative Cognition, and Their Interactive Effects on Depressed Mood
}

\author{
Jeffrey A. Ciesla \\ Kent State University
}

\author{
John E. Roberts \\ University at Buffalo, State University of New York
}

\begin{abstract}
Response styles theory posits that rumination represents a trait vulnerability to depression. Recent evidence has suggested that rumination predicts changes in depression more strongly among individuals with high levels of negative cognition. Three studies evaluated this model of interactive vulnerabilities. Study 1 provided empirical support for the distinction between rumination and negative cognitive content. The next 2 studies investigated the interactive model in the laboratory. Study 2 randomly assigned participants to either ruminate or distract following a sad mood induction. This study found that rumination was more strongly associated with dysphoria among individuals who report high levels of negative cognition. Similarly, Study 3 found that rumination and negative cognition interact to predict changes in dysphoria across a no-task delay period following a sad mood induction. These studies provide support for an interactive model in which rumination amplifies the deleterious effects of negative cognition.
\end{abstract}

Keywords: rumination, dysfunctional attitudes, self-esteem, attributional style
The response styles theory of depression (Nolen-Hoeksema, 1991) proposes that individuals differ in their reaction to negative mood states and that these differences affect the eventual course of these moods. More specifically, rumination, defined as "behaviors and thoughts that focus one's attention on one's depressive symptoms and on the implication of these symptoms" (NolenHoeksema, 1991, p. 569) may serve to maintain and/or exacerbate depression. In contrast, responses that serve to distract individuals from their depression were posited to alleviate feelings of sadness. Numerous investigations have supported the predictions of the response styles theory. In particular, rumination has been shown to be prospectively related to severity of depression (Just \& Alloy, 1997; Nolan, Roberts, \& Gotlib, 1998; Nolen-Hoeksema \& Morrow, 1991; Nolen-Hoeksema, Parker, \& Larson, 1994), duration of depressive symptoms (Nolen-Hoeksema, Morrow, \& Fredrickson, 1993), and recovery from depression (Kuehner \& Weber, 1999; Schmaling, Dimidjian, Katon, \& Sullivan, 2002), as well as the onset of new episodes of depression (Nolen-Hoeksema, 2000; Spasojevic \& Alloy, 2001).

Morrow and Nolen-Hoeksema (1990) proposed multiple mechanisms through which rumination may affect depression, including the possibility that rumination enhances "the effects of existing maladaptive cognitive styles by bringing maladaptive cognitions to mind more often" (p. 520). It is important to note that this hypothesis does not suggest that rumination creates such maladaptive cognitions, but rather that rumination amplifies their effect. This

Jeffrey A. Ciesla, Department of Psychology, Kent State University; John E. Roberts, Department of Psychology, University at Buffalo, State University of New York.

Correspondence concerning this article should be addressed to Jeffrey A. Ciesla, Department of Psychology, Kent State University, P.O. Box 5190, Kent, OH 44242-0001. E-mail: jciesla@kent.edu perspective hypothesizes that ruminative response styles and maladaptive cognitive schemata may represent two vulnerabilities to depression that moderate each others' relation with depression. In many ways, this perspective is similar to priming theories of depression (Miranda \& Persons, 1988; Segal \& Ingram, 1994), which suggest that negative cognitive content may lie dormant or inactive unless activated by another process.

In prior research of this interactive rumination model (Ciesla \& Roberts, 2002), we proposed that the cognitive process-cognitive content distinction may be useful in understanding the relationship between rumination and negative cognition. We proposed that rumination could be thought of as a type of cognitive process or, put another way, that rumination concerns how one thinks. Broadly speaking, rumination is characterized by a style of thought (i.e., repetitive or obsessive) rather than the specific content of that thought. One could ruminate about work, vacation plans, or even the weather.

In contrast, we have conceptualized negative cognition as dealing with content, or concerning what one thinks. Multiple theories of depression have been proposed that posit that vulnerable individuals espouse negative beliefs or make specific maladaptive attributions that place them at risk for depressive disorder. In Beck's (1967) theory of depression, these include rigid and unrealistic beliefs, particularly of the self, world, and future. In the helplessness theory of depression (Abramson, Seligman, \& Teasdale, 1978), these involve stable, global, and internal attributions for negative events. Borrowing from a number of theoretical perspectives (Beck, 1967; Brown \& Harris, 1978), we have also included negative beliefs about the self and low self-esteem under this rubric, although we note that this particular type of cognition is sometimes conceptualized as a symptom of depression rather than a vulnerability to it (Abramson, Metalsky, \& Alloy, 1989; Roberts \& Monroe, 1994) or as both a vulnerability and a symptom (Bernet, Ingram, \& Johnson, 1993). 
To further articulate the interactive rumination model, consider Figure 1. In this model, a relation between negative cognition and depression is depicted by the solid black line, in which such cognition represents a vulnerability to disorder. An additional dotted line suggests that this relation may be reciprocal in nature, such that increases in depression may increase the accessibility of negative cognition. Ruminative thought is depicted in the center of this figure, operating as a catalyst. The presence of rumination would increase the influence of negative cognition on depression and in turn increase the influence of depression on cognitive content. Thus, a maladaptive cycle would be maintained or even accelerated; ruminating on negative beliefs worsens depression and ruminating on depression reinforces and supports the negative beliefs. In this way, rumination and negative cognitive content operate in a synergistic and mutually self-perpetuating manner in generating depression. Such a model would imply that clinical improvement may be achieved by intervening with either rumination or negative cognitive content. Interrupting rumination would disengage the engine that drives the cycle, whereas modifying negative cognitive content would set the cycle on a new and more adaptive course. In either case, the maladaptive recycling of negative thinking would decelerate, ultimately leading to remission of the episode.

In our earlier research (Ciesla \& Roberts, 2002), we investigated rumination, negative cognition, and their interaction as predictors of response to group treatment for depression. Although there were no significant main effects of rumination on three different measures of negative cognition, two of three Rumination $\times$ Cognition interaction terms were significant predictors of treatment response. ${ }^{1}$ Among individuals with high levels of negative cognition, rumination was associated with a poorer prognosis. Interestingly, among those with low levels of negative cognition, rumination was associated with a better response to treatment, although this effect was only marginally significant in subsequent unpublished simple slope analyses (Ciesla \& Roberts, 2006). This finding is consistent with the perspective that under certain circumstances, self-directed thought may be adaptive rather than maladaptive (see Teasdale \& Hilary, 2004; Watkins \& Teasdale, 2004). Although this investigation showed the importance of the interaction of these factors in recovery from depression, it does not

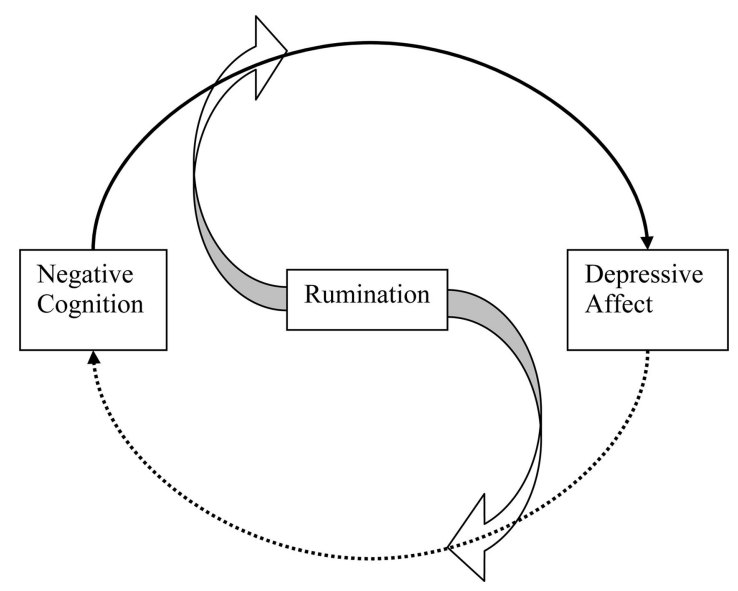

Figure 1. Schematic representation of the interactive rumination model. address whether this interaction is involved in the etiology of depression.

Robinson and Alloy (2003) found evidence for an interaction of rumination and negative cognition in a prospective study of 148 university students who were followed for a 2.5 -year period. The authors found that stress-reactive rumination, the tendency to ruminate in response to stressful life events, was more strongly predictive of future episodes of major and hopeless depression ${ }^{2}$ among individuals who reported high levels of negative content than among individuals who reported low levels. Depressive rumination also showed this interaction in predicting hopeless depression but not major depressive episodes.

Thus, two prior studies (Ciesla \& Roberts, 2002; Robinson \& Alloy, 2003) have provided evidence that rumination and negative cognition interact to predict changes in depression. However, the literature investigating the main effects of rumination on depression has not relied solely on prospective investigations of depressive disorder. Instead, this literature has been strengthened by laboratory studies examining the effects of rumination under more controlled situations and in predicting changes in depressive affect. For example, multiple studies have found that among dysphoric individuals, a ruminative task leads to the persistence of depressive mood, whereas a distracting task leads to decreases in such mood (e.g., Lyubomirsky \& Nolen-Hoeksema, 1993, 1995; NolenHoeksema \& Morrow, 1993).

These experimental studies demonstrate that when people are instructed to ruminate, dysphoric mood is maintained and/or exacerbated. However, these studies do not speak to whether individuals vary in their tendency to ruminate when given the opportunity to do so and whether such trait rumination is associated with the maintenance or exacerbation of dysphoric mood. Conway, Csank, Holm, and Blake (2000) demonstrated that individuals who receive high scores on a self-report measure of rumination evidence greater dysphoric affect following a negative mood induction than those who receive low scores, but only after a brief no-task delay period during which they are able to spontaneously ruminate. Thus, high levels of self-reported trait rumination appear related to self-initiated rumination that contributes to the persistence of dysphoric mood.

Thus, two distinct methods have been used to investigate rumination in the laboratory: (a) experimental manipulation of rumination and (b) measurement of trait rumination as a predictor of change during a delay period. These two methods have their respective strengths and limitations. The manipulation of a participant's response to sadness is admittedly artificial, and findings may not generalize; this design cannot address whether natural tendencies to ruminate would have similar effects. Furthermore, the study's hypotheses are relatively transparent. In contrast, the second methodology addresses some of these concerns but relies heavily on the validity of self-report measures of rumination to predict individuals' actual ruminative responses during the delay

\footnotetext{
${ }^{1}$ Significant interactions with the Dysfunctional Attitudes Scale and Rosenberg Self-Esteem Scale were observed, but not with the Attributional Style Questionnaire. See Study 1's Method section for a description of these measures.

${ }^{2}$ See Abramson et al. (1989) for a review of and criteria for the construct of hopeless depression.
} 
period. It is difficult to determine whether high trait ruminators really did ruminate during the delay. In general, we become more confident of the internal validity of a study when rumination is manipulated and more confident of external validity when rumination is measured.

The present article reports a series of three studies testing this interactive model of negative cognition and rumination. Study 1 empirically examined the validity of the rumination-negative cognition-depression distinction. Using confirmatory factor analysis, we asked whether multiple measures of these constructs would conform to a hypothesized model of three correlated factors and whether more parsimonious models with fewer factors could better explain their covariance. Studies 2 and 3 tested the interactive model using the two aforementioned laboratory methodologies. These studies were designed and implemented simultaneously, and their order of presentation is therefore somewhat arbitrary.

\section{Study 1: Discriminant Validity of Rumination, Negative Cognition, and Depressive Symptoms}

\section{Method}

\section{Participants}

Participants were recruited from introductory psychology classes at the University at Buffalo for a study on personality, mood, and media. No inclusion or exclusion criteria were used; students signed up to participate in exchange for course credit. Individuals who signed up for participation were randomly assigned to either Study 2 or Study 3 (discussed below), which were run concurrently. Of the 263 students who participated in either study, 138 were female and 125 were male, and the majority (84\%) were in either their freshman or sophomore year of college. The median age was 19 (interquartile range $=2.0$ ). The majority of participants were Caucasian (59\%), followed by Asian (24\%), and the remainder (17\%) identified themselves as African American, Hispanic, or other.

\section{Measures}

Rosenberg Self-Esteem Scale (RSE; Rosenberg, 1979). The RSE is a widely used 10-item scale that measures participants' global self-worth. Participants rate their agreement with 10 statements about their general self-concept (e.g., "On the whole, I am satisfied with myself" and "At times, I think I am no good at all"). Previous studies have found that the RSE demonstrates good test-retest reliability ( $r \mathrm{~s}=.85$ to .88 ; see Rosenberg, 1979). In the present sample, coefficient alpha was .90 .

Dysfunctional Attitudes Scale (DAS; Weissman \& Beck, 1978). The DAS was used in addition to the RSE as a second measure of negative cognition. This 40-item scale assesses the endorsement of rigid and perfectionistic beliefs thought to be common among depressed individuals (e.g., "People will probably think less of me if I make a mistake" and "I cannot be happy unless most people I know admire me"). In the present sample, coefficient alpha was .91 .

Attributional Style Questionnaire (ASQ; Peterson et al., 1982). A third index of negative cognition, the ASQ, asks participants about their attributions or explanations for six hypothetical events.
Individuals report the likelihood that the hypothetical events were caused by the self (Internal subscale), would have lasting negative consequences (Stable subscale), and would have negative implications for other domains of the participant's life (Global subscale). The scores on each of these subscales are then summed to create one overall composite, with higher scores indicative of a more negative attributional style. ${ }^{3}$ In the present sample, coefficient alpha was .70.

Beck Depression Inventory, Second Edition (BDI-II; Beck, Steer, \& Brown, 1996). The BDI-II was used to measure participants' levels of depressive symptomatology. Separate Cognitive-Affective and Somatic subscales were identified from the work of Storch, Roberti, and Roth (2004). This widely used 21-item scale asks the respondent to indicate the presence and severity of symptoms of depression during the past 2 weeks. In the present sample, coefficient alpha for the full measure was .91 .

Response Styles Questionnaire-Rumination Scale (RSQ; Nolen-Hoeksema \& Morrow, 1991). The 33-item version of the RSQ asks participants to report their typical responses to depressed mood. This questionnaire consists of a 22-item Rumination scale and an 11-item Distraction scale. Only the Rumination scale was examined in this study. On this measure, participants indicate the frequency with which they engage in a number of different responses (e.g., think about a recent situation wishing it had gone better, think about how sad you feel) to depressed mood on a 4-point Likert scale.

Rumination on Sadness Scale (RSS; Conway et al., 2000). Similar to the RSQ, the RSS is a 13-item self-report measure of the tendency to ruminate in response to negative moods. This measure has demonstrated high internal consistency $(\alpha=.91)$ and a unifactorial structure in previous research (Conway et al., 2000) Coefficient alpha in the current study was .93 .

\section{Procedure}

Participants were run through the study protocol individually in a quiet laboratory room. All individuals completed each of the self-report measures and were randomized to then continue in either Study 2 or Study 3 (no participant completed both Studies 2 and 3).

\section{Results and Discussion}

Confirmatory factor analysis was used to test the hypothesized relation among each of the measures. Initially, the hypothesized model with three correlated factors was evaluated: (a) Rumination, measured using the RSQ and RSS; (b) Negative Content, measured using the RSE, DAS, and ASQ; and (c) Depression, measured with the Affective-Cognitive and Somatic subscales of the BDI-II. As we recruited an unselected sample of undergraduates, these variables were generally normally distributed with a minor skew in which there were slightly more adaptive than maladaptive scores.

The hypothesized model fit the data very well, $\chi^{2}(11, N=$ $263)=25.49, p<.01$, Incremental Fit Index $($ IFI $)=.99$, TuckerLewis index $=.97$, comparative fit index $=.99$, root mean square

\footnotetext{
${ }^{3}$ Each of the ASQ subscales was explored in both Studies 1 and 2. The pattern of findings did not appreciably differ across subscale, thus only the results of the overall composite are presented.
} 
error of approximation $=.07$. Each of the factor loadings were substantial and highly significant (all $\lambda \mathrm{s}>.4$ and $p \mathrm{~s}<.001$ ). The correlation between Rumination and Negative Content was .72 $(p<.001)$, whereas it was $.68(p<.001)$ between Rumination and Depression and $76(p<.001)$ between Negative Content and Rumination.

Further analyses explored the discriminant validity of the original hypothesized model. Alternative nested models examined whether measures of hypothesized constructs could be collapsed into a single factor. For example, we tested a model in which the measures of negative cognition and rumination all loaded on a single latent variable, retaining depression as a second correlated factor. This model provided a significantly poorer fit to the data, $\Delta \chi^{2}(2)=74.61, p<.001$. Similarly, models in which the depression indicators were combined with the measures of negative cognition, $\Delta \chi^{2}(2)=39.59, p<.001$, or with the measures of rumination, $\Delta \chi^{2}(2)=139.13, p<.001$, also resulted in significantly poorer model fits. Thus, the results of Study 1 supported the theoretical distinctions among rumination, negative cognitive content, and depression. Gender did not moderate any of these analyses in multigroup model comparisons.

\section{Study 2: An Experimental Manipulation of Rumination}

After establishing the convergent and discriminant validity of the indices of our constructs, we turned our attention to evaluating the possible interaction of rumination and negative cognition. In Study 2, an experimental manipulation of rumination and distraction was used to investigate the hypothesis that individuals' response to a rumination task would be dependent on preexisting levels of negative cognition. Dysphoric individuals who report high levels of negative cognition were hypothesized to find an internally focused task more aversive than those who report lower levels of negative cognition. However, we hypothesized that a distraction task would alleviate dysphoria regardless of the presence of negative cognition. Such a task, in turning attention outward, would be expected to render the presence or absence of negative cognition irrelevant. Thus, we hypothesized an interaction between preexisting negative cognition and experimental task (rumination vs. distraction) in predicting changes in dysphoric affect.

\section{Method}

\section{Participants}

After participating in Study 1, 133 students were randomly assigned to continue in Study 2; 73 were female and 60 were male, and the majority $(87 \%)$ were in either their freshman or sophomore year of college. The median age was 19 years (interquartile range $=2.0)$. The majority of participants were Caucasian $(56 \%)$, followed by Asian (29\%), and the remainder (15\%) identified themselves as African American, Hispanic, or other.

\section{Measures}

The three measures of negative cognition (DAS, RSE, and ASQ) that were completed by participants in Study 1 were used as predictors in Study 2, as was the BDI-II.
The Multiple Affect Adjective Checklist-Revised (MAACL-R; Zuckerman \& Lubin, 1985) is a widely used, 132-item measure of various affective states. Using the results of a factor analysis of this measure (Gotlib \& Meyer, 1986), the 10 items with the strongest loading on a depressive affect factor were selected for inclusion in a modified questionnaire. Participants responded to the various items on a 5-point scale ranging from 1 (not at all) to 5 (extremely). To distract participants from the true purpose of the study and to disguise the repeated administration of the measure, 27 items reflecting various personality traits (e.g., responsible, extraverted, conscientious) were added to these original 10, resulting in a total of 37 items. Using these items, three different forms of the questionnaire were used as repeated measures of dysphoric affect. These questionnaires used the same items and response scale, but varied in the order of item presentation, style of font, size of font, borders, and even page length (one to two pages). The results were three very different looking questionnaires containing identical items. The modified 10-item depressive affect scale, which we refer to as the modified MAACL, or MAACL-M, demonstrated excellent internal consistency across each form $(\alpha \mathrm{s}=$ $.90, .92$, and .91 , respectively).

\section{Procedure}

Participants were run through the study protocol individually in a quiet laboratory room. After completing the measures described in Study 1 and an initial MAACL-M (Time 1), all individuals watched a 5-min movie clip depicting a woman's death from cancer and her family's reaction. This clip has been shown to elicit a sad mood both in pilot work for this study and in previous published research (Halberstadt \& Niedenthal, 1997). Participants were told, "This film clip is approximately 5 minutes long. Please sit quietly, watch the clip, and [the experimenter] will return after it is complete." Once this clip finished, participants completed a second MAACL-M (Time 2).

According to random assignment, participants were then instructed to engage in either a ruminative $(N=67)$ or a distracting $(N=66)$ cognitive task. These tasks were developed by Morrow and Nolen-Hoeksema (1990) and have been used repeatedly in prior experimental studies of rumination (Lyubomirsky et al., 1998, 1999, 2003; Lyubomirsky \& Nolen-Hoeksema, 1993, 1995; Vickers \& Vogeltanz-Holm, 2003). In these tasks, participants were provided with sheets of paper with sentences printed on them. They were instructed to "read each item slowly and quietly to yourself. As you read the items, use your imagination and concentration to focus your mind on each of these things. Spend a few moments visualizing and concentrating on each item." Furthermore, individuals were instructed that should they complete all of the sentences, they should start over again with the first and continue until the experimenter returned to the room and asked them to stop. The ruminative and distracting tasks differed only in the content of the sentences. The ruminative task consisted of selfand emotion-focused sentences such as "Think about the level of motivation you feel right now" and "Think about why you react the way you do." In contrast, the distracting task consisted of statements such as "Think about the shadow of a stop sign" and "Think about and imagine a ship slowly crossing the Atlantic." The experimenter asked the participant to begin and left the room for $8 \mathrm{~min}$. At the end of this time, the experimenter returned and asked the participant to complete a final MAACL-M (Time 3). 
Last, the participant listened to an upbeat piece of music to reverse the effects of the sad mood induction. All participants were then debriefed and assessed to make sure that the effects of the video mood induction had been alleviated.

\section{Results and Discussion}

\section{Preliminary Analyses}

Initial analyses revealed no significant association of study variables with age, gender, year in school, or religion. However, minority students had significantly higher DAS scores, $t(131)=$ 2.11, $p<.05$, as well as higher MAACL-M scores at Time 2, $t(131)=2.41, p<.05$. Therefore, ethnic minority status coded as a binary variable was used as a covariate in the analyses using this combination of measures. The mood induction procedure was effective in increasing levels of dysphoric affect from Time 1 to Time 2 (Time $1 M=17.0$; Time $2 M=20.3$ ), $t(132)=4.44, p<$ .001 . Zero-order correlations of the study variables are presented in Table 1.

\section{Regression Analyses}

Multiple linear regression with forced stepwise entry was used to test the main effects of the rumination task and negative cognition, as well as their interaction (the Rumination $\times$ Negative Cognition moderation model) in predicting persistence of dysphoric mood. In three regression models, each measure of negative cognition was examined separately, with Time 3 MAACL-M as the dependent variable. The first step of the models added Time 2 MAACL-M to control for pretask affect. Step 2 then added a dummy-coded variable for experimental task along with an index of negative cognition. The final step added the corresponding Task $\times$ Negative Cognition interaction term. Continuous variables were centered before forming interaction terms to reduce nonessential multicollinearity (see Aiken \& West, 1991.)

Self-esteem. The first model, investigating self-esteem, revealed that higher levels of posttask dysphoria were associated with low self-esteem $(\beta=-0.40, p r=-.44, p<.001)$ and experimental task $(\beta=0.21, p r=.27, p<.01)$. Individuals with lower self-esteem and those assigned to the rumination task were more dysphoric at Time 3. Of most theoretical relevance, these main effects were qualified by a significant Task $\times$ RSE interaction term $(\beta=-0.17, p r=-.22, p<.05$; see Table 2$)$. The form of this interaction was further explored graphically (see Figure 2) by computing predicted values at each of the two levels of the experimental manipulation and with simple slopes analyses (Aiken \& West, 1991), in which the dichotomous values for the two tasks are separately entered into the full regression equation, resulting in two distinct lines that can be tested for significance. Results indicated that the effect of self-esteem was larger when conditioned for the rumination task $(\beta=-0.57, p r=-.46, p<.001)$ compared with the distraction task $(\beta=-0.23, p r=-.20, p<$ $.05)$.

Dysfunctional attitudes. Because of the significant effect of ethnic minority status on both DAS scores and MAACL-M scores, this variable was controlled for in the analyses examining dysfunctional attitudes, although inclusion of this covariate did not appreciably change any of the results. This model revealed that higher levels of posttask dysphoria were associated with high levels of dysfunctional attitudes $(\beta=0.26, p r=.28, p<.01)$ as well as experimental task $(\beta=0.22, p r=.26, p<.01)$. Individuals with higher levels of dysfunctional attitudes and those assigned to the rumination task were more dysphoric at Time 3 . These main effects were qualified by a significant Task $\times$ DAS interaction term $(\beta=0.15, p r=.19, p<.05$; see Table 2$)$. This interaction is plotted in Figure 3. Simple slope analyses showed that higher DAS scores were associated with higher levels of dysphoric affect when conditioned at the rumination condition $(\beta=0.43, p r=.33$, $p<.001)$, but were not significantly associated with dysphoric affect when conditioned at the distraction condition $(\beta=0.12$, $p r=.11, p=.23$ ).

Attributional style. The regression model including attributional style was examined last. In this model, higher levels of posttask dysphoria were associated with higher total ASQ scores $(\beta=0.16, p r=.18, p<.05)$ and the experimental task $(\beta=0.23$, $p r=.27, p<.01)$. Individuals with more negative attributional styles and those assigned to the rumination task were more dysphoric at Time 3 . The Task $\times$ ASQ interaction term was not a significant predictor of Time 3 affect controlling for Time 2 affect $(\beta=-0.04, p r=-.05, p=.60)$.

Although preexisting levels of dysphoric affect were already controlled in the above analyses, we explored the BDI-II as a covariate in these models owing to the significant overlap of depressive symptoms with our other predictors. It is possible, for example, that individuals with higher levels of depressive symptoms could evidence more pervasive negative affect in the study,

Table 1

Means, Standard Deviations, and Zero-Order Correlations: Study 2

\begin{tabular}{|c|c|c|c|c|c|c|c|c|c|c|}
\hline Measure & 1 & 2 & 3 & 4 & 5 & 6 & 7 & $M$ & $S D$ & $\alpha$ \\
\hline 1. RSE & - & & & & & & & 38.96 & 7.74 & .91 \\
\hline 2. DAS & $-.62^{* * *}$ & - & & & & & & 123.31 & 31.27 & .92 \\
\hline 3. ASQ & $-.29^{* *}$ & $.47^{* * *}$ & - & & & & & 74.38 & 11.25 & .62 \\
\hline 4. BDI-II & $-.78^{* * * *}$ & $.60^{* * * *}$ & $.30^{* *}$ & - & & & & 11.04 & 8.88 & .90 \\
\hline 5. MAACL-M, T1 & $-.65^{* * *}$ & $.53^{* * *}$ & $.32^{* * * *}$ & $.68^{* * * *}$ & - & & & 17.01 & 7.23 & .90 \\
\hline 6. MAACL-M, T2 & $-.40^{* * *}$ & $.36^{* * * *}$ & $.20^{*}$ & $.47^{* * * *}$ & $.43^{* * *}$ & - & & 20.27 & 8.55 & .92 \\
\hline 7. MAACL-M, T3 & $-.54^{* * * *}$ & $.39^{* * * *}$ & $.22^{*}$ & $.61^{* * * *}$ & $.61^{* * * *}$ & $.50^{* * * *}$ & - & 14.53 & 5.83 & .91 \\
\hline
\end{tabular}

Note. $\quad$ RSE $=$ Rosenberg Self-Esteem Scale; DAS = Dysfunctional Attitudes Scale; ASQ = Attributional Style Questionnaire; BDI-II = Beck Depression Inventory 2nd ed.; MAACL-M = Multiple Affect Adjective Checklist, modified; T1 = Time 1; T2 = Time 2; T3 = Time 3.

${ }^{*} p<.05 .{ }^{* *} p<.01 .{ }^{* * * *} p<.001$. 
Table 2

Self-Esteem, Dysfunctional Attitudes, and Attributional Style Regression Models: Study 2

\begin{tabular}{|c|c|c|c|c|c|c|c|c|c|c|c|c|}
\hline \multirow[b]{2}{*}{ Step and predictor } & \multicolumn{4}{|c|}{ Self-esteem } & \multicolumn{4}{|c|}{ Dysfunctional attitudes } & \multicolumn{4}{|c|}{ Attributional style } \\
\hline & $\beta$ & $p r$ & $t$ & $\Delta R^{2}$ & $\beta$ & $p r$ & $t$ & $\Delta R^{2}$ & $\beta$ & $p r$ & $t$ & $\Delta R^{2}$ \\
\hline Step 1 & & & & $.25^{* * * *}$ & & & & $.25^{\text {**** }}$ & & & & $.25^{* * * *}$ \\
\hline T2 MAACL-M & 0.50 & .50 & $6.52^{* * *}$ & & 0.50 & .49 & $6.52^{* * * *}$ & & 0.50 & .50 & $6.52^{* * * *}$ & \\
\hline Step 2 & & & & $.18^{* * *}$ & & & & $.10^{* * * *}$ & & & & $.07^{* * *}$ \\
\hline T2 MAACL-M & 0.35 & .39 & $4.75^{* * *}$ & & 0.42 & .43 & $5.40^{* * *}$ & & 0.48 & .49 & $6.40^{* * *}$ & \\
\hline Task & 0.21 & .26 & $3.11^{* *}$ & & 0.22 & .26 & $3.08^{* *}$ & & 0.23 & .27 & $3.13^{* * *}$ & \\
\hline Cognition & -0.40 & -.44 & $-5.51^{* * *}$ & & 0.26 & .28 & $3.35^{* *}$ & & 0.16 & .18 & $2.09^{*}$ & \\
\hline Step 3 & & & & $.03^{* *}$ & & & & $.02^{*}$ & & & & .00 \\
\hline T2 MAACL-M & 0.23 & .29 & $3.40^{* * *}$ & & 0.42 & .44 & $5.53^{* * * *}$ & & 0.48 & .49 & $6.37^{* * *}$ & \\
\hline Task & 0.22 & .31 & $3.65^{* * *}$ & & 0.22 & .27 & $3.14^{* *}$ & & 0.23 & .27 & $3.13^{* *}$ & \\
\hline Cognition & -0.40 & -.44 & $-5.57^{* * *}$ & & 0.27 & .39 & $3.59^{* * *}$ & & 0.16 & .18 & $2.12^{*}$ & \\
\hline Task $\times$ Cognition & -0.17 & -.22 & $-2.57^{*}$ & & 0.15 & .19 & $2.17^{*}$ & & -0.04 & -.05 & -0.52 & \\
\hline
\end{tabular}

Note. T2 MAACL-M = Time 2 Multiple Affect Adjective Check List, modified; Task = experimental task; Cognition = corresponding index of negative cognition.

${ }^{*} p<.05 .{ }^{* *} p<.01 .{ }^{* * *} p<.001$.

and effects of the predictor variables could be attributed to their initial correlation with the BDI-II. Controlling for the BDI-II did not affect the pattern of findings in any of the prior analyses. Finally, gender was explored as a moderator of each of these previously mentioned tests. None of the analyses revealed a significant higher order gender interaction.

\section{Study 3: Recovery From a Dysphoric Mood Induction}

In contrast to the experimental manipulation of rumination in Study 2, our third study was designed to investigate naturally occurring rumination. This study used a modification of the laboratory paradigm developed by Conway and colleagues (2000). Here, self-reported trait rumination is measured initially, followed by a dysphoric mood induction, and then a no-task delay period is provided to allow participants the opportunity to spontaneously ruminate. High self-reported ruminators are expected to engage in more ruminative thought during this period than low self-reported ruminators. Furthermore, the moderation model hypothesizes that negative cognition would interact with this tendency to ruminate in predicting changes in dysphoric affect. Specifically, trait rumina-

\section{Task X RSE}

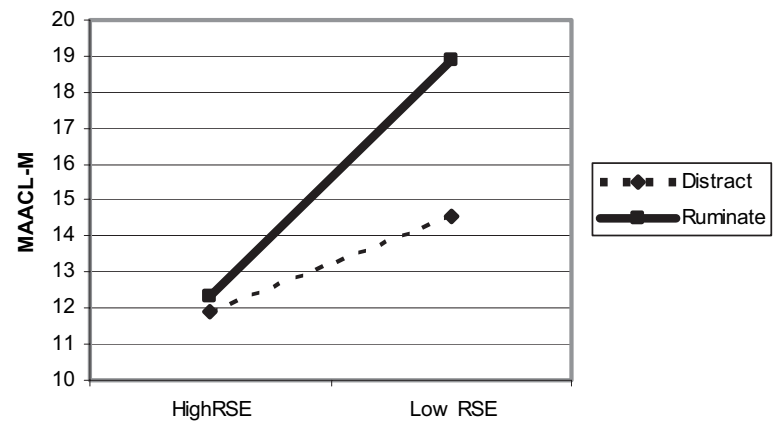

Figure 2. Interaction of experimental task and self-esteem in predicting Time 3 affect, Study 2. RSE = Rosenberg Self-Esteem Scale; MAACL$\mathrm{M}=$ Multiple Affect Adjective Checklist, modified. tion would have a stronger effect among individuals reporting greater negative cognition.

\section{Method}

\section{Participants}

Of the 126 students who participated in Study 3, 63 were female and 63 were male, and the majority $(83 \%)$ were in either their freshman or sophomore year of college. The distribution of age in the sample was positively skewed with a median of 19 years (interquartile range $=2.0$ ). The majority of participants were Caucasian (64\%), followed by Asian (18\%), and the remainder $(18 \%)$ identified themselves as African American, Hispanic, Native American, or other.

\section{Measures}

The three measures of negative cognition (DAS, RSE, and ASQ) and two measures of rumination (RSS and RSQ) that were completed by participants in Study 1 were used as predictors in Study 2, as was the BDI-II. In addition, to explore the possibility that various forms of rumination may interact differently with negative cognition, Brooding and Reflective Pondering subscales of the RSQ were extracted following the work of Treynor, Gonzalez, and Nolen-Hoeksema (2003). Finally, the MAACL-M was again used to assess dysphoric affect.

\section{Procedure}

Participants were run through the study protocol individually in a quiet laboratory room. They were told that the study was designed to assess individual reactions to different video and audio stimuli. Individuals first completed a baseline series of assessments, including the RSQ, RSS, RSE, DAS, ASQ, BDI-II, and the Time 1 MAACL-M. After this assessment, all individuals completed the 5-min sad mood induction described in Study 1. Following this, participants completed a Time 2 MAACL-M. After this was finished, the experimenter entered the room and explained in a flustered manner that the next task would involve listening to 
a piece of music, but that as a result of unforeseen circumstances the materials were not ready and it would take a few minutes to prepare (if pressed, the experimenter explained that the last audiotape had been damaged in the tape player and a new audio clip was being dubbed off of a compact disk). The experimenter apologized for the delay and assured the individual that her or his participation would still end on time. The experimenter then explained that the study was designed for the tasks to be completed back-to-back, so the participant should just "sit quietly" and wait for the experimenter to return. The participant was then left alone for $8 \mathrm{~min}$. After this time passed, the experimenter returned with an audiotape player with headphones, asked the participant to complete a Time 3 MAACL-M, and had the participant listen to an upbeat piece of music to reverse the effects of the sad mood induction. All participants were then debriefed and interviewed to ensure that the effects of the original sad mood induction had been reversed and to check whether the participant had believed the cover story for the delay period. No participant guessed that the delay period was an intended part of the experiment.

\section{Results and Discussion}

\section{Preliminary Analyses}

Preliminary analyses revealed that no demographic factor (i.e., age, gender, year in school, racial or ethnic minority, religion) was significantly associated with any of the study variables. A dependent-samples $t$ test revealed that the mood induction was successful in increasing MAACL-M scores (Time $1 M=16.7$; Time $2 M=19.8), t(125)=4.07, p<.001$. As expected, MAACL-M depression scores on average decreased during the no-activity delay period relative to scores at Time 2 (Time $3 M=$ 14.7), $t(125)=7.91, p<.001$.

The zero-order correlations among the study variables can be seen in Table 3. The correlation between the two measures of rumination was substantial $(r=.81, p<.001)$, and therefore a single rumination score was derived by summing participants' standardized scores on the RSQ and RSS.

\section{Regression Analyses}

Multiple linear regression with forced stepwise entry was then used to test the main effects of rumination and negative cognition, as well as their interactions (the Rumination $\times$ Negative Cognition moderation model) in predicting persistence of dysphoric mood. The same analytic strategy followed in Study 1 was used in this study, with a separate regression model run for each measure of negative cognition. Specifically, in these regression analyses, the dependent variable was Time 3 MAACL-M. The first step of these regression models involved the addition of Time 2 MAACL-M as a covariate. The second step included the rumination index score as well as a measure of negative cognition. ${ }^{4}$ The third step included the interaction term of the two variables entered in the second step. All variables were centered before forming interaction terms to reduce nonessential multicollinearity.

Self-esteem. The model examining self-esteem revealed a significant main effect of self-esteem $(\beta=-0.40, p r=-.39, p<$ $.001)$ such that individuals reporting lower self-esteem had higher levels of dysphoria after the no-task delay. No significant main
Task X DAS

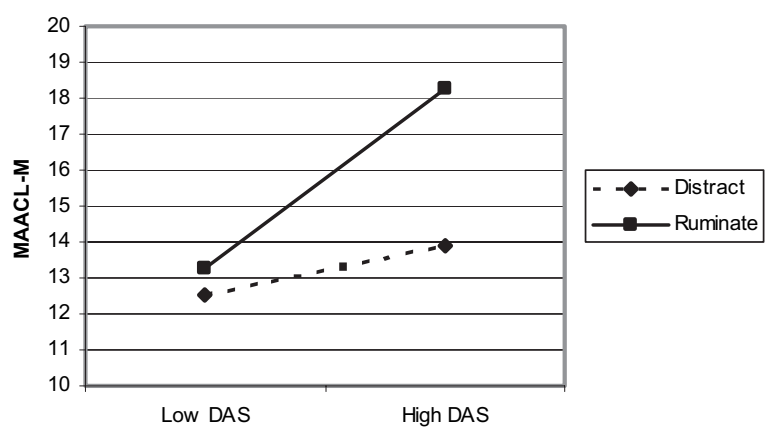

Figure 3. Interaction of experimental task and dysfunctional attitudes in predicting Time 3 affect, Study 2. DAS = Dysfunctional Attitudes Scale; MAACL-M = Multiple Affect Adjective Checklist, modified.

effect of rumination was detected $(\beta=0.08, p r=.08, p=.40$ ). Of most theoretical relevance, these results were qualified by a significant Rumination $\times$ Self-Esteem interaction term $(\beta=$ $-0.21, p r=-.27, p<.01$; see Table 4). As can be seen in Figure 4 , the form of this interaction revealed that although on average higher levels of trait rumination were predictive of higher levels of postdelay dysphoria, this effect was stronger for those with low levels of self-esteem. Simple slope analyses (Aiken \& West, 1991) indicated that self-esteem was not a statistically significant predictor of residual dysphoria after the no-delay task when conditioned 1 standard deviation below the mean on rumination $(\beta=-0.12$, $p r=-.11, p=.24)$, whereas it predicted residual increases in dysphoria when conditioned at 1 standard deviation above the mean $(\beta=-0.54, p r=-.46, p<.001)$.

Follow-up analyses revealed that this interaction was also present when the Brooding subscale from the RSQ was used ( $\beta=$ $-0.21, p r=-.28, p<.01)$, whereas the Reflective Pondering subscale yielded a marginal trend $(\beta=-0.12, p r=-.16, p=$ $.08)$. The form of both of these interactions mirrored the results using our full measure of rumination.

Dysfunctional attitudes. The dysfunctional attitudes model resulted in a significant main effect of rumination $(\beta=0.23, p r=$ $-.23, p<.05)$ such that higher levels of trait rumination were associated with higher postdelay dysphoria. A marginal trend was found suggesting that higher levels of dysfunctional attitudes were also associated with depressive affect $(\beta=0.17, p r=.17, p=$ .06). These effects were qualified by a significant Rumination $\times$ DAS interaction $(\beta=0.22, p r=.27, p<.01$; see Table 4$)$. The form of this interaction is plotted in Figure 5, which shows that higher levels of rumination were associated with dysphoric affect only among participants with high DAS scores. Simple slopes analyses found that dysfunctional attitudes were significantly associated with increases in residual dysphoria when conditioned 1 standard deviation above the mean on rumination $(\beta=0.32, p r=$ $.28, p<.01$ ), but were not associated with dysphoria when rumination was conditioned 1 standard deviation below the mean $(\beta=-0.07, p r=-.06, p=.54)$.

\footnotetext{
${ }^{4}$ The RSQ and RSS were examined separately in similar analyses. The results were essentially unchanged, and the composite findings are presented in the interest of brevity.
} 
Table 3

Means, Standard Deviations, and Zero-Order Correlations: Study 3

\begin{tabular}{|c|c|c|c|c|c|c|c|c|c|c|c|c|}
\hline & 1 & 2 & 3 & 4 & 5 & 6 & 7 & 8 & 9 & $M$ & $S D$ & $\alpha$ \\
\hline 1. RSQ & - & & & & & & & & & 48.73 & 13.30 & .93 \\
\hline 2. RSS & $.81^{* * * *}$ & - & & & & & & & & 30.55 & 11.26 & .92 \\
\hline 3. RSE & $-.58^{* * * *}$ & $-.61^{* * * *}$ & - & & & & & & & 39.47 & 7.57 & .90 \\
\hline 4. DAS & $.53^{* * * *}$ & $.55^{* * * *}$ & $-.48^{* * * *}$ & - & & & & & & 124.03 & 28.34 & .90 \\
\hline 5. ASQ & $.35^{* * * *}$ & $.38^{* * * *}$ & $-.35^{* * *}$ & $.43^{* * *}$ & - & & & & & 73.06 & 12.07 & .74 \\
\hline 6. BDI-II & $.59^{* * * *}$ & $.63^{* * * *}$ & $-.66^{* * *}$ & $.62^{* * * *}$ & $.38^{* * * *}$ & - & & & & 10.14 & 9.16 & .92 \\
\hline 7. MAACL-M, T1 & $.60^{* * * *}$ & $.60^{* * * *}$ & $-.60^{* * *}$ & $.49^{* * *}$ & $.28^{* *}$ & $.74^{* * * *}$ & - & & & 16.73 & 7.76 & .94 \\
\hline 8. MAACL-M, T2 & $.42^{* * * *}$ & $.35^{* * * *}$ & $-.37^{* * * *}$ & $.42^{* * * *}$ & .13 & $.46^{* * * *}$ & $.45^{\text {**** }}$ & - & & 19.78 & 8.30 & .92 \\
\hline 9. MAACL-M, T3 & $.46^{* * * *}$ & $.44^{* * * *}$ & $-.58^{* * * *}$ & $.46^{* * * *}$ & $.20^{*}$ & $.63^{* * * *}$ & $.74^{* * * *}$ & $.54^{* * * *}$ & - & 14.69 & 6.38 & .92 \\
\hline
\end{tabular}

Note. $\quad$ RSQ $=$ Response Styles Questionnaire; RSS = Rumination on Sadness Scale; RSE = Rosenberg Self-Esteem Scale; DAS = Dysfunctional Attitudes Scale; ASQ = Attributional Style Questionnaire; BDI-II = Beck Depression Inventory 2nd edition; MAACL-M = Multiple Affect Adjective Checklist, modified; $\mathrm{T} 1=$ Time $1 ; \mathrm{T} 2=$ Time $2 ; \mathrm{T} 3=$ Time 3 .

${ }^{*} p<.05 .^{* * *} p<.01 .{ }^{* * * *} p<.001$.

Analysis using the RSQ subscales revealed a marginal trend for the DAS $\times$ Brooding interaction $(\beta=0.14, p r=.17, p=.06)$, whereas the DAS $\times$ Reflective Pondering interaction was nonsignificant $(\beta=0.12, p r=.14, p=.12)$. Although neither of these interactions were statistically significant, the form of the interactions was similar to that observed with the full measure of rumination.

Attributional style. The attributional style model revealed a significant main effect of rumination $(\beta=0.29, p r=.30, p<.01)$ such that higher levels of trait rumination were associated with residual increases in postdelay dysphoria, and the ASQ was not significantly predictive of postdelay affect $(\beta=0.04, p r=.04$, $p=.65)$. Finally, the Rumination $\times$ ASQ interaction term was also not significant $(\beta=0.06, p r=.07, p=.59)$. Neither the Brooding $(\beta=0.02, p r=.01, p=.95)$ nor the Reflective Pondering ( $\beta=0.32, p r=.10, p=.29)$ RSQ subscales yielded significant interactions with the ASQ.

Once again, we reran the above analyses on an exploratory basis, controlling for the BDI-II. Controlling for depressive symptoms in addition to preexisting depressive affect did not affect the pattern of findings. Similarly, none of the previous effects were qualified by a significant higher order gender interaction.

\section{General Discussion}

This article reports the results of two laboratory studies investigating the hypothesis that the impact of rumination on depressive affect varies as a function of the nature and degree of negative cognitive content. The results of Study 1 provided the foundation for testing this model by demonstrating the discriminant validity of the constructs. The results of Study 2 supported the interactive model, showing that the impact of a rumination task was moderated by levels of negative cognition. Study 3 provided evidence that trait rumination and negative cognition significantly predict recovery from a dysphoric mood state. These studies showed that in addition to the main effects of rumination and negative cognition, these vulnerabilities interacted with and amplified each others' effects.

These results may help explain why the majority of published studies have supported the response styles theory of depression,

Table 4

Self-Esteem, Dysfunctional Attitudes, and Attributional Style Regression Models: Study 3

\begin{tabular}{|c|c|c|c|c|c|c|c|c|c|c|c|c|}
\hline \multirow[b]{2}{*}{ Step and predictor } & \multicolumn{4}{|c|}{ Self-esteem } & \multicolumn{4}{|c|}{ Dysfunctional attitudes } & \multicolumn{4}{|c|}{ Attributional style } \\
\hline & $\beta$ & $p r$ & $t$ & $\Delta R^{2}$ & $\beta$ & $p r$ & $t$ & $\Delta R^{2}$ & $\beta$ & $p r$ & $t$ & $\Delta R^{2}$ \\
\hline Step 1 & & & & $.29^{* * * *}$ & & & & $.29^{* * *}$ & & & & $.29^{* * *}$ \\
\hline T2 MAACL-M & 0.54 & .54 & $7.18^{* * * *}$ & & 0.54 & .54 & $7.18^{* * * *}$ & & 0.54 & .54 & $7.18^{* * * *}$ & \\
\hline Step 2 & & & & $.18^{* * * *}$ & & & & $.10^{* * *}$ & & & & $.08^{* *}$ \\
\hline T2 MAACL-M & 0.36 & .41 & $4.97^{* * * *}$ & & 0.38 & .40 & $4.78^{* * * *}$ & & 0.42 & .44 & $5.36^{* * * *}$ & \\
\hline Rumination & 0.08 & .08 & 0.89 & & 0.23 & .23 & $2.58^{*}$ & & 0.29 & .30 & $3.48^{* * *}$ & \\
\hline Cognition & -0.40 & -.39 & $-4.69^{* * * *}$ & & 0.17 & .17 & $1.88^{\dagger}$ & & 0.04 & .04 & 0.46 & \\
\hline Step 3 & & & & $.04^{* *}$ & & & & $.04^{* *}$ & & & & .00 \\
\hline T2 MAACL-M & 0.37 & .43 & $5.20^{* * * *}$ & & 0.39 & .42 & $5.03^{* * * *}$ & & 0.43 & .44 & $5.40^{* * * *}$ & \\
\hline Rumination & 0.07 & .08 & 0.85 & & 0.20 & .21 & $2.36^{*}$ & & 0.28 & .28 & $3.21^{* * *}$ & \\
\hline Cognition & -0.34 & -.34 & $-4.00^{* * * *}$ & & 0.13 & .13 & 1.43 & & 0.04 & .04 & 0.46 & \\
\hline RSQ $\times$ Cognition & -0.21 & -.27 & $3.11^{* * *}$ & & 0.22 & .27 & $3.03^{* *}$ & & 0.06 & .07 & 0.77 & \\
\hline
\end{tabular}

Note. T2 MAACL-M = Time 2 Multiple Affect Adjective Check List, modified; Rumination = Composite of Response Styles Questionnaire and Rumination on Sadness Scale; Cognition = corresponding index of negative cognition; RSQ = Response Styles Questionnaire.

${ }^{\dagger} p<.01 .{ }^{*} p<.05 .{ }^{* * *} p<.01 .{ }^{* * * *} p<.001$. 
yet notable exceptions exist (Bagby et al., 1999; Lara, Klein, \& Kasch, 2000). The moderation model suggests that the relation between rumination and depression is conditional on the level of negative cognition in a given sample. As such, owing to sampling variability, investigators likely draw samples that differ in their mean level of negative cognition, resulting in observed associations between rumination and depression that vary. Analyses involving the rumination subscales suggested that the moderation hypothesis can be observed with different aspects of ruminative thought. This is particularly noteworthy given that previous work (Treynor et al., 2003) had suggested that the main effects of brooding were stronger than the effects of reflective pondering. Although the authors suggested that reflection may be adaptive over time, our results suggest that this may be dependent on the presence of negative cognition. In individuals with high levels of negative cognition, otherwise innocuous contemplation may create a downward cognitive-affective spiral. This perspective is similar to that of Joorman, Dkane, and Gotlib (2006, p. 277), who proposed that among depressed individuals pondering and brooding "might easily perpetuate each other, thus blurring the distinction between adaptive and maladaptive cognitive styles." Stated more generally, among individuals prone to depression, attempts to engage in reflective pondering may inevitably result in brooding.

Support for the moderation model was observed in both Studies 2 and 3, but only when the index of negative cognition was either the RSE or the DAS. The pattern of significant findings with measures of self-esteem and dysfunctional attitudes, combined with a lack of significance with a measure of negative attributional style, is remarkably consistent with prior research (Ciesla \& Roberts, 2002). In contrast, Robinson and Alloy (2003) found a significant Risk $\times$ Rumination interaction when high risk was defined as having a high score on both the DAS and a measure of attributional style. However, because these questionnaires were used in conjunction to define cognitive vulnerability in this study, it cannot be determined whether these findings were a result of the influence of dysfunctional attitudes or attributional style. Thus, studies have found support for significant interactions of rumination that were specific to self-esteem and dysfunctional attitudes, but similar results have not been obtained for attributional style. One explanation for this pattern of findings is that attributional style may contribute to depression in a different manner. Specifi-

Trait Rumination X RSE

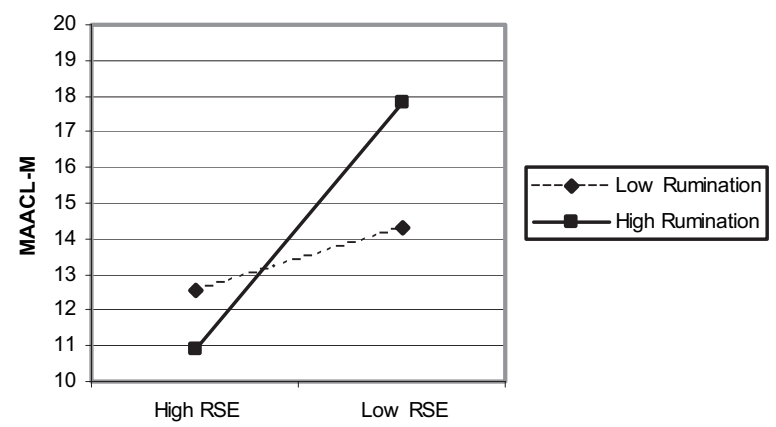

Figure 4. Interaction of rumination and self-esteem in predicting Time 3 affect, Study 3. RSE = Rosenberg Self-Esteem Scale; MAACL-M = Multiple Affect Adjective Checklist, modified.
Trait Rumination X DAS

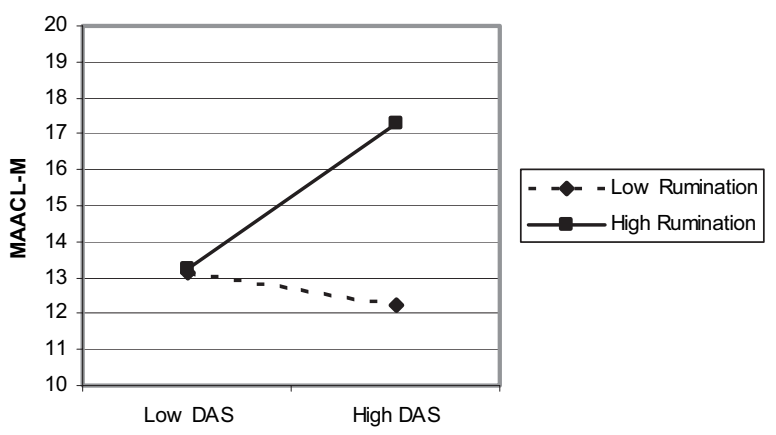

Figure 5. Interaction of rumination and dysfunctional attitudes in predicting Time 3 affect, Study 3. DAS = Dysfunctional Attitudes Scale; MAACL-M = Multiple Affect Adjective Checklist, modified.

cally, attributional style involves explanations for stressful life events rather than core, general beliefs. Because rumination involves internal self-focus, it may have limited impact on thoughts about external events. Another explanation for the lack of significant findings involving attributional style involves the lower internal consistencies observed with our six-situation version of the ASQ compared with the RSE and DAS. This lower reliability limits the magnitude of the relationship the ASQ could feasibly have with a dependent variable, and this limitation is further compounded in an interaction term with another imperfect measure.

The results of the present studies along with previous work (Ciesla \& Roberts, 2002; Robinson \& Alloy, 2003) demonstrate that individuals who are prone to engage in rumination are at heightened risk for depressive affect and symptomatology, although only if they also have high levels of negative cognition. Although these two vulnerabilities interact, our studies also confirmed significant main effects. That is, the presence of either one of these vulnerabilities on its own is a risk factor for dysphoric affect, although this risk may be amplified under circumstances in which the other vulnerability is elicited. For example, a highly ruminative person who has high self-esteem may function well in general. However, the moderation model suggests that this individual may become depressed in the face of personally invalidating experiences such as a job loss, academic failure, or romantic rejection that threatens self-esteem. Similarly, a relatively nonreflective individual who rarely engages in rumination but who has high levels of dysfunctional attitudes may function well in general. However, this individual may be at risk for depression under circumstances in which active or distractive coping is less effective, such as following the death of a loved one or the diagnosis of a serious disease.

These findings may have implications for psychological intervention. Roadblocks in psychotherapy are common. Therapy can become mired while one is struggling to modify the content of severely negative cognition or conversely by the sheer repetitive and obsessive self-focus exhibited by some patients. In such cases, our model would posit that changing the focus of treatment from one vulnerability to the other could free the logjam. For example, when therapy is "stuck" when addressing low self-esteem, it may be wise to shift the emphasis to interrupting rumination, and later 
return to addressing low self-esteem when the patient is able address those issues in a less perseverative and unproductive manner. In the absence of rumination, low self-esteem may be more malleable as it is no longer continually amplified. As such, therapists may cease to view rumination and low self-esteem as independent problems but rather as part of a larger, integrated, and maladaptive cognitive system requiring a flexible treatment approach.

These findings suggest a number of different possibilities for future research. First, investigations are needed in which the impact of differing forms of negative cognition can be differentiated. Furthermore, prior research has suggested that rumination interacts with life stress (Schwartz \& Koenig, 1996), and thus we hypothesize that the impact of the Rumination $\times$ Negative Cognition interaction may be stronger among individuals experiencing high levels of life stress. Other work has begun to investigate different forms of ruminative thought, such as experiential versus analytical rumination (Watkins \& Teasdale, 2004) and reflective versus ruminative self-focus (Teasdale \& Hilary, 2004). In addition, another perspective on the interplay between rumination and negative cognitive content has been articulated by Spasojevic and Alloy (2001). In this model, rumination is posited to represent a common mechanism through which a variety of risk factors (including negative cognitive content) affect depression. Future longitudinal research is needed to further elucidate the direction and nature of the causal relationships among rumination, negative content, and depression. In addition, gender did not moderate the observed interactive effects. That is, the interactive model appears to apply equally to men and women. However, it is important to note that the present studies concern changes in dysphoric affect rather than depressive disorder. Future studies are needed to examine the role of gender in this model in the context of clinically significant depressive symptomatology.

Finally, the moderation model may have interesting implications for the study of disorders and affects other than depression. $\mathrm{Nu}$ merous psychiatric disorders (e.g., eating disorders and anxiety disorders) and affective states (e.g., anger and anxiety) are accompanied by repetitive, obsessive, or ruminative thought. Future research is needed to explore whether various types of cognitive content amplify the effects of rumination on these other forms of disorder and affect. We hypothesize that rumination itself is a nonspecific vulnerability that can fuel various types of negative cognitive content, leading to specific psychological outcomes. For example, rumination in combination with a distorted body image may lead to eating disorders; rumination in combination with fears of embarrassment may lead to social phobia, and so forth. In addition, little is known regarding whether a process similar to rumination may influence the duration of positive affective states and whether such an effect may be moderated by other factors. The potential for rumination to influence a wider range of clinical states is a promising area for future research.

We note that, relative to the observed main effects in both studies, the interaction terms accounted for a relatively small portion of variance $(2 \%-4 \%)$ in the dependent variable. However, small effect sizes are often observed with interaction terms, due in part to their natural collinearity with the main effects but also because of the compounding of measurement error inherent in forming product terms. Describing this phenomenon, Aiken and West (1991, p. 163) cautioned, "When reliability [of the predictors and criterion] drops to $80 \%$, the variance accounted for by the interaction decreases by 50\%." Thus, effect sizes of product terms based on imperfect measures must be understood as significant underestimations of the size of the true effects.

Some limitations of the present research are worth noting. First, we did not have a manipulation check associated with our laboratory tasks; no attempt was made to ascertain whether participants truly ruminated or distracted in Study 2 or 3. Thus, we can only infer the behavior of participants during these times. Although we constructed very different forms of our affect measure to attempt to avoid practice effects and the influence of demand characteristics, their influence cannot be ruled out. However, we think it unlikely that participants would be able to guess that our studies were predicting a statistical interaction and even more unlikely that they would be able to artificially produce one. One final limitation concerns the use of university students as our participants. Future investigations using clinical populations are needed.

\section{References}

Abramson, L. Y., Metalsky, G. I., \& Alloy, L. B. (1989). Hopelessness depression: A theory-based subtype of depression. Psychological Review, 96, 358-372.

Abramson, L. Y., Seligman, M. E. P., \& Teasdale, J. (1978). Learned helplessness in humans: Critique and reformulation. Journal of Abnormal Psychology, 87, 49-74.

Aiken, L. S., \& West, S. G. (1991). Multiple regression: Testing and interpreting interactions. Newbury Park, CA: Sage.

Bagby, R. M., Rector, N. A., Segal, Z. V., Joffe, R. T., Levitt, A. J., Kennedy, S. H., \& Levitan, R. D. (1999). Rumination and distraction in major depression: Assessing response to pharmacological treatment. Journal of Affective Disorders, 55, 225-229.

Beck, A. T. (1967). Depression: Clinical, experimental, and theoretical aspects. New York: International Universities Press.

Beck, A. T., Steer, R. A., \& Brown, G. K. (1996). Manual for the Beck Depression Inventory-II. San Antonio, TX: Psychological Corporation.

Bernet, C. Z., Ingram, R. E., \& Johnson, B. R. (1993). Self-esteem. In C. G. Costello (Ed.), Symptoms of depression (pp. 141-159). New York: Wiley.

Brown, G. W., \& Harris, T. O. (1978). Social origins of depression: A study of psychiatric disorder in women. New York: Free Press.

Ciesla, J. A., \& Roberts, J. E. (2002). Self-directed thought and response to treatment for depression: A preliminary investigation. Journal of Cognitive Psychotherapy: An International Quarterly, 16, 435-453.

Ciesla, J. A., \& Roberts, J. E. (2006). [Unpublished simple slope analyses of data from Ciesla and Roberts, 2002]. Unpublished analyses, Vanderbilt University, Nashville, TN.

Conway, M., Csank, P. A. R., Holm, S. L., \& Blake, C. K. (2000). On individual differences in rumination on sadness. Journal of Personality Assessment, 75, 404-425.

Gotlib, I. H., \& Meyer, J. P. (1986). Factor analysis of the multiple adjective check list: A separation of positive and negative affect. Journal of Personality and Social Psychology, 49, 233-242.

Halberstadt, J. B., \& Niedenthal, P. M. (1997). Emotional state and the use of stimulus dimensions in judgment. Journal of Personality and Social Psychology, 72, 1017-1033.

Joorman, J., Dkane, M., \& Gotlib, I. H. (2006). Adaptive and maladaptive components of rumination? Diagnostic specificity and relation to depressive biases. Behavior Therapy, 37, 269-280.

Just, N., \& Alloy, L. B. (1997). The response styles theory of depression: Tests and extension of the theory. Journal of Abnormal Psychology, 106, 221-229.

Kuehner, C., \& Weber, I. (1999). Responses to depression in unipolar 
depressed patients: An investigation of Nolen-Hoeksema's response styles theory. Psychological Medicine, 29, 1323-1333.

Lara, M. E., Klein, D. N., \& Kasch, K. (2000). Psychosocial predictors of the short-term course and outcome of major depression: A longitudinal study of a nonclinical sample with recent-onset episodes. Journal of Abnormal Psychology, 109, 644-650.

Lyubomirsky, S., Caldwell, N.D., \& Nolen-Hoeksema, S. (1998). Effects of ruminative and distracting responses to depressed mood on retrieval of autobiographical memories. Journal of Personality and Social Psychology, 75, 166-177.

Lyubomirsky, S., Kasri, F., \& Zehm, K. (2003). Dysphoric rumination impairs concentration on academic tasks. Cognitive Therapy and Research, 27, 309-330.

Lyubomirsky, S., \& Nolen-Hoeksema, S. (1993). Self-perpetuating properties of dysphoric rumination. Journal of Personality and Social Psychology, 65, 339-349.

Lyubomirsky, S., \& Nolen-Hoeksema, S. (1995). Effects of self-focused rumination on negative thinking and interpersonal problem solving. Journal of Personality and Social Psychology, 69, 176-190.

Lyubomirsky, S., Tucker, K. L., Caldwell, N. D., \& Berg, K. (1999). Why ruminators are poor problem solvers: Clues from the phenomenology of dysphoric rumination. Journal of Personality and Social Psychology, 77, 1041-1060.

Miranda, J., \& Persons, J. B. (1988). Dysfunctional attitudes are moodstate dependent. Journal of Abnormal Psychology, 97, 76-79.

Morrow, J., \& Nolen-Hoeksema, S. (1990). Effects of responses to depression on the remediation of depressive affect. Journal of Personality and Social Psychology, 58, 519-527.

Nolan, S. A., Roberts, J. E., \& Gotlib, I. H. (1998). Neuroticism and ruminative response style as predictors of change in depressive symptoms. Cognitive Therapy and Research, 22, 445-455.

Nolen-Hoeksema, S. (1991). Responses to depression and their effects on the duration of depressive episodes. Journal of Abnormal Psychology, 100, 569-582.

Nolen-Hoeksema, S. (2000). The role of rumination in depressive disorders and mixed anxiety/depressive symptoms. Journal of Abnormal Psychology, 109, 504-511.

Nolen-Hoeksema, S., \& Morrow, J. (1991). A prospective study of depression and posttraumatic stress symptoms after a natural disaster: The 1989 Loma Prieta earthquake. Journal of Personality and Social Psychology, 61, 115-121.

Nolen-Hoeksema, S., \& Morrow, J. (1993). Effects of rumination and distraction on naturally occurring depressed mood. Cognition and Emotion, 7, 561-570.

Nolen-Hoeksema, S., Morrow, J., \& Fredrickson, B. L. (1993). Response styles and the duration of episodes of depressed mood. Journal of Abnormal Psychology, 102, 20-28.

Nolen-Hoeksema, S., Parker, L. E., \& Larson, J. (1994). Ruminative coping with depressed mood following loss. Journal of Personality and Social Psychology, 67, 92-104.

Peterson, C., Semmel, A., von Baeyer, C., Abramson, L. Y., Metalskiy, G. I., \& Seligman, M. E. P. (1982). The attributional style questionnaire. Cognitive Therapy and Research, 6, 287-300.

Roberts, J. E., \& Monroe, S. M. (1994). A multidimensional model of self-esteem in depression. Clinical Psychology Review, 14, 161-181.

Robinson, M. S., \& Alloy, L. B. (2003). Negative cognitive styles and stress-reactive rumination interact to predict depression: A prospective study. Cognitive Therapy and Research, 27, 275-291.

Rosenberg, M. (1979). Conceiving the self. New York: Basic Books.

Schmaling, K. B., Dimidjian, S., Katon, W., \& Sullivan, M. (2002). Response styles among patients with minor depression and dysthymia in primary care. Journal of Abnormal Psychology, 111, 350-356.

Schwartz, J. A. J., \& Koenig, L. J. (1996). Response styles and negative affect among adolescents. Cognitive Therapy and Research, 20, 13-36.

Segal, Z. V., \& Ingram, R. E. (1994). Mood priming and construct activation in tests of cognitive vulnerability to unipolar depression. Clinical Psychology Review, 14, 663-695.

Spasojevic, J., \& Alloy, L. B. (2001). Rumination as a common mechanism relating depressive risk factors to depression. Emotion, 1, 25-37.

Storch, E. A., Roberti, J. W., \& Roth, D. A. (2004). Factor structure, concurrent validity, and internal consistency of the Beck Depression Inventory-2nd ed. in a sample of college students. Depression and Anxiety, 19, 187-189.

Teasdale, J. D., \& Hilary, G. A. C. (2004). Ruminative self-focus and autobiographical memory. Personality and Individual Differences, 36, 1933-1943

Treynor, W., Gonzalez, R., \& Nolen-Hoeksema, S. (2003). Rumination reconsidered: A psychometric analysis. Cognitive Therapy and Research, 27, 247-259.

Vickers, K. S., \& Vogeltanz-Holm, N. D. (2003). The effects of rumination and distraction tasks on psychophysiological responses and mood in dysphoric and nondysphoric individuals. Cognitive Therapy and Research, 27, 331-348.

Watkins, E., \& Teasdale, J. D. (2004). Adaptive and maladaptive self-focus in depression. Journal of Affective Disorders, 82, 1-8.

Weissman, A. N., \& Beck, A. T. (1978, November). Development and the validation of the Dysfunctional Attitude Scale. Paper presented that the meeting of the Association for the Advancement of Behavior Therapy, Chicago.

Zuckerman, M., \& Lubin, B. (1985). The Multiple Affect Adjective Check List revised. San Diego, CA: EdITS.

Received July 7, 2006

Revision received April 2, 2007

Accepted April 3, 2007 